\title{
How to Define Acute Liver Failure Patients with Pre-Existing Liver Disease without Signs of Cirrhosis
}

\author{
Aline Gottlieb ${ }^{a}$ Maren Kottmann ${ }^{b}$ Paul Mankab Sotiria Bedrelib \\ Johannes Hadem ${ }^{a, b}$ Lars Bechmann ${ }^{a}$ Jan-Peter Sowa ${ }^{a, b}$ Guido Gerken ${ }^{b}$ \\ Ali Canbay ${ }^{a}$ \\ ${ }^{a}$ Department of Gastroenterology, Hepatology und Infectious Diseases, University Hospital, Otto-von-Guericke \\ University, Magdeburg, Germany; ${ }^{b}$ Department for Gastroenterology and Hepatology, Center for Internal Medicine, \\ University Hospital, University Duisburg-Essen, Essen, Germany
}

\section{Keywords}

Acute-on-chronic · Outcome $\cdot$ Clinical management

\section{Abstract}

Background: The definition of acute liver failure (ALF) usually implies no previous liver injury. Though, some patients admitted to liver transplantation centers with the diagnosis of ALF are obese or have diabetes. Elevated liver enzymes were not recorded previously, and no signs of cirrhosis or prior decompensation of the liver function were ever present. Still, these patients differ from the "typical" ALF-patient. Goals: In this study, we aimed to confirm acute-on-chronicliver failure (AOCLF) in patients diagnosed with ALF and to identify possible differences between ALF and AOCLF. Study: Patients were retrospectively recruited from all patients admitted to the University Hospital Essen with diagnosis of ALF between 2008 and 2015. Data of 163 patients were evaluated, resulting in a reclassification of 32 patients as $\mathrm{AO}$ CLF (remaining ALF: 131). Demographic and clinical data as well as serum parameters, including cell death markers, were correlated with clinical outcome. Results: Patients with AOCLF were significantly older, had a higher body mass index
(๑) 2018 S. Karger AG, Basel
(BMI), and were more often male. The cause for liver failure in these patients differed significantly from patients who had an actual ALF. Significant differences were also found for serum liver enzymes. Outcome of patients did not differ between AOCLF and ALF. Though, lower BMI and MELD and higher AST and GLDH were predictors for a beneficial outcome. Conclusion: AOCLF is still commonly misdiagnosed as ALF. While clinical outcome does not significantly differ between ALF and AOCLF, risk factors for adverse outcome may significantly differ between these entities.

(c) 2018 S. Karger AG, Basel

\section{Introduction}

Acute liver failure (ALF) is the final common result of severe hepatocyte injury [1]. ALF itself is one of the most dangerous complications in hepatology, with a high rate of mortality $[2,3]$ and immediate requirement of intensive care [1]. When patients are admitted with the diagnosis of ALF to a tertiary care or liver transplant center, a thorough examination is necessary to identify the underlying cause of ALF and the severity of the situation [4]. Initial diag-
KARGER

E-Mail karger@karger.com www.karger.com/ddi
Prof. Dr. Ali Canbay

Head of the Department of Gastroenterology

Hepatology and Infectious Diseases, Otto-von-Guericke University

Leipzigerstr. 44, DE-39120 Magdeburg (Germany)

E-Mail ali.canbay@med.ovgu.de 
Table 1. Principal differences in the definition and diagnostic criteria of acute-on-chronic liver failure between APASL and EASL definitions

\begin{tabular}{lll}
\hline Parameter & APASL definition & EASL-CLIF definition \\
\hline Total bilirubin & $5 \mathrm{mg} / \mathrm{dL}$ or more & $12 \mathrm{mg} / \mathrm{dL}$ or more \\
INR & 1.5 or more & 2.5 or more \\
Hepatic encephalopathy & Any grade & Only grade III and IV \\
Ascites & May be present & Not included \\
Duration between insult and ACLF & 4 week & Not defined \\
Acute event - sepsis & No & Yes \\
Acute event - variceal bleeding & No unless producing jaundice and & Yes \\
Extra-hepatic organ involvement & coagulopathy defining ACLF & No \\
What is chronic disease & Chronic liver disease with/without & Only cirrhosis, including those \\
& only compensated cirrhosis & with prior decompensation \\
\hline \multirow{2}{*}{ INR, international normalized ratio; ACLF, acute-on-chronic liver failure. } \\
\hline
\end{tabular}

nostic tests often reveal either newly diagnosed or already known diabetes, overweight or other previously present but yet undiagnosed risk factors, that is, considerable ethanol consumption, that can affect the normal liver function in these patients. If pre-admission records indicate elevated liver enzymes in such a patient, this should be considered as a warning sign for possible pre-existing liver damage [5]. Pre-existing liver damage per definition excludes the diagnosis of ALF, even if no signs of liver cirrhosis (such as spider naevi, ascites, splenomegaly) are present. In these cases, the correct diagnosis would be acute-on-chronic-liver failure (AOCLF). It is estimated that $25 \%$ of the world population has non-alcoholic fatty liver disease [6], which undoubtedly constitutes pre-existing liver disease, but is often disregarded or goes unnoticed. Though, there are conflicting results for the impact of non-alcoholic fatty liver disease on outcome of an acute injury or surgical procedure in the liver [7-11].

AOCLF is considered to be clinically distinct from ALF as well as decompensated cirrhosis $[12,13]$. In the past years, varying definitions of this disease entity have been proposed $[14,15]$. However, no common definition has been agreed upon so far. Currently there are two main approaches to define AOCLF proposed by Asian-Pacific or European researchers (Table 1). In the Asian-Pacific definition, AOCLF is a clinical syndrome manifesting as acute and severe hepatic dysfunction resulting from multiple insults [16], 1 pre-existing chronic and 1 acute. The preexisting liver damage can be present with or without cirrhosis, though cirrhosis must be compensated. In contrast, Jalan et al. [14] proposed that AOCLF is an acute decompensation of patients with liver cirrhosis, including cases with previous decompensation events. Nevertheless, after an acute insult, AOCLF can lead either to a chronic injury or a liver failure with or without reversibility.

In the present study, we aimed to identify clinically relevant differences between patients with an ALF compared to patients suffering from AOCLF, excluding any patients with liver cirrhosis. In particular, we analyzed if there are any early identifiers to separate ALF from AOCLF patients and if the clinical course or outcome were different between these two entities. One crucial observation was that the initial diagnosis of ALF had to be corrected to AOCLF in a relevant number of patients to accurately reflect the presence of pre-existing liver disease. This implies that chronic liver disease still remains unnoticed in many cases.

\section{Methods}

\section{Patients}

In this retrospective single-center study, 163 Caucasian patients were included (99 female, 64 male patients), who were admitted with an initial diagnosis of ALF between November 2008 and November 2015. These patients were retrospectively divided into two groups: people suffering from an actual ALF and those with a newly diagnosed pre-existing liver damage without cirrhosis and no prior history of decompensation. Due to the retrospective nature of the study, the institutional review board waived requirement for written informed consent. All procedures adhered to the requirements of the institutional review board and the current version of the Declaration of Helsinki.

\section{Laboratory Parameters}

Serum enzyme concentrations [aspartate aminotransferase (AST), alanine aminotransferase (ALT), glutamate dehydrogenase (GLDH), AP, GGT, bilirubin, creatinine] were determined on a Bayer Advia 120 clinical chemistry analyzer (Bayer, Leverkusen, 
Table 2. Demographic and clinical data of the patient cohort at admission

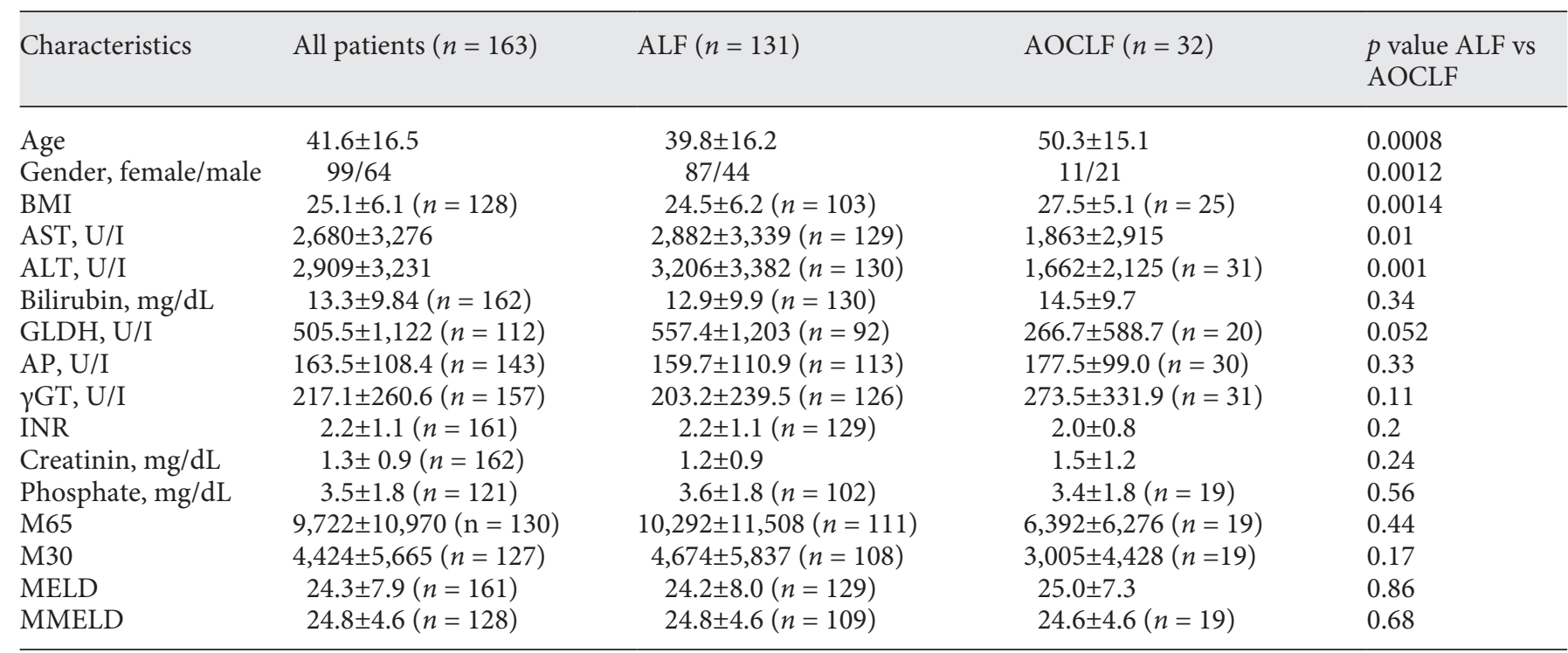

ALF, acute liver failure; AOCLF, acute-on-chronic liver failure; BMI, body mass index; AST, aspartate aminotransferase; ALT, alanine aminotransferase; GLDH, glutamate dehydrogenase; AP, alcalic phosphatase; $\gamma \mathrm{GT}, \gamma$-glutamyltransferase; INR, international normalized ratio; M65, surrogate cell death marker (epithelial cells); M30, surrogate apoptosis marker (epithelial cells); MELD, model for end-stage liver disease; MMELD, CK18-modified MELD.

Germany), and international normalized ratio (INR) was determined/calculated on the Sysmex CA 5000 coagulation analyzer (Sysmex Europe, Norderstedt, Germany).

\section{Body Mass Index}

Patients' body weights were determined upon admission, if possible. The presence of ascites was excluded using ultrasound. Body mass index (BMI) was calculated according to the following formula:

$$
\text { BMI = body weight }(\mathrm{kg}) \times \text { height }^{2}\left(\mathrm{~m}^{2}\right)
$$

\section{Cell Death Markers and Cytokeratin 18-Based Modification of} $M E L D$

The surrogate serum markers for overall epithelial cell death (M65, TecoMedical, Sissach, Switzerland) and apoptosis (M30, TecoMedical) were assessed by enzyme-linked immunosorbent assay according to manufacturer's instructions. Absorption at 450 nm was detected on a FLUOStar ${ }^{\circledR}$ Omega (BMG Labtech, Ortenberg Germany). We have previously described a modification of the MELD score, incorporating serum M65 concentrations, termed CK18-modified MELD (MMELD) [17]. The MMELD achieved higher sensitivity to predict the survival of ALF patients in the respective cohort. Based on the measurements in the current patient cohort, MMELD was calculated for 128 patients (ALF, AOCLF).

\section{Statistics}

For normally distributed parameters, data are given as mean \pm $\mathrm{SD}$, and Student's $t$ test was used to calculate differences between groups. Mann-Whitney test was performed when data was not normally distributed or non-parametric. Kruskal-Wallis and
Dunns tests were performed as post-hoc tests for not-normally distributed continuous variables. Fisher's exact test was applied to test for differences in dichotomous variables. Statistical significance was considered at $p \leq 0.05$. Analyses were performed with Prism, version 5 (GraphPad Software Inc., La Jolla, CA, USA).

\section{Results}

\section{Patients with an AOCLF are Significantly Older and} have a Higher BMI

Demographic and clinical data of the whole patient cohort are given in Table 2. In some cases, not all parameters were collected and the actual number of patients for each parameter and group is given in parenthesis. Individual medical histories, clinical and laboratory data recorded at our center were thoroughly screened and analyzed to either confirm diagnosis of ALF or reclassify patients as AOCLF. Patients were defined as AOCLF, when pre-existing liver disease was retrospectively identified in individuals with an initial diagnosis of ALF. In total, 32 of 163 ALF patients were re-defined as AOCLF. The chronic liver diseases present in these patients are given in online supplementary Table 1 (for all online suppl. material, see www.karger.com/doi/10.1159/000492869). 
Fig. 1. Serum transaminases are lower in AOCLF compared to ALF. Serum concentrations of AST (a) and ALT (b) were significantly lower in patients with AOCLF in comparison to ALF patients. Shown are scatter dot plots with mean and SEM. Statistical significance was determined by Mann-Whitney U Test.

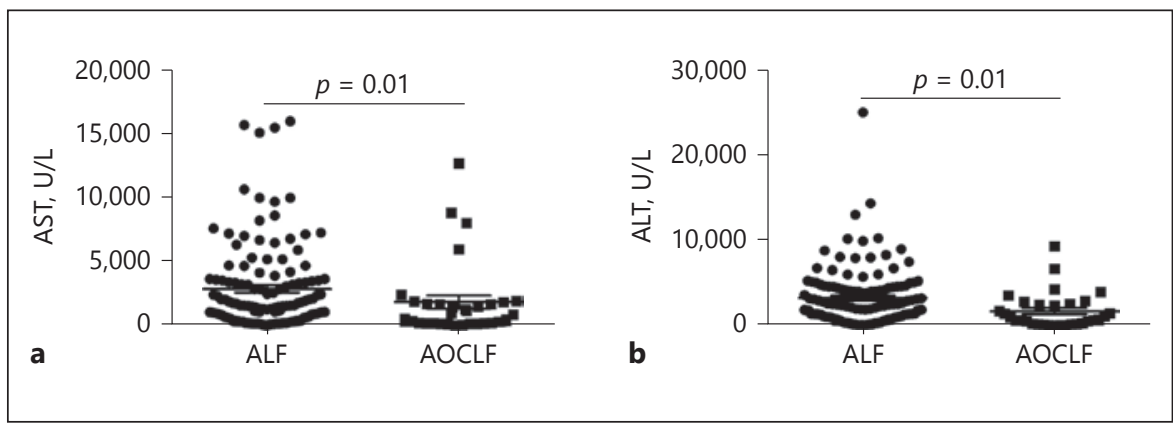

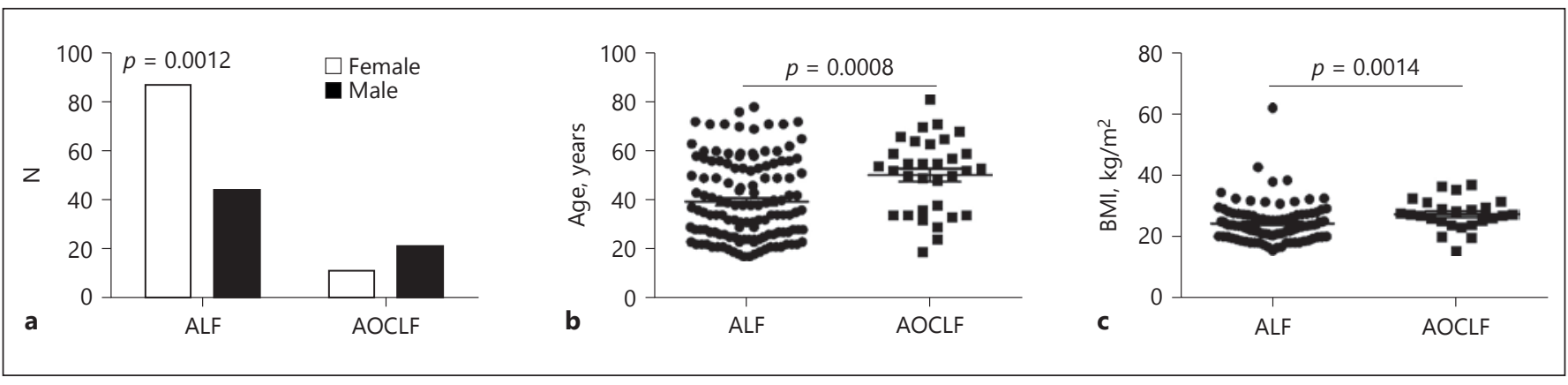

Fig. 2. Serum transaminases are lower in acute-on-chronic liver failure (AOCLF) compared to acute liver failure (ALF). Serum concentrations of AST (a) and ALT (b) were significantly lower in patients with AOCLF in comparison to ALF patients, whereas the

Table 3. Etiologies of acute insult leading to liver failure in ALF or AOCLF

\begin{tabular}{lcc}
\hline Etiology & ALF, $n(\%)$ & AOCLF, $n(\%)$ \\
\hline Pharmacological/toxic & $48(36.6)$ & $4(12.5)$ \\
Acute viral infection & $36(27.5)$ & $12(37.5)$ \\
Autoimmune hepatitis & $16(12.2)$ & $3(9.3)$ \\
Unknown & $21(16.0)$ & $4(12.5)$ \\
Other (rare) causes & $10(7.6)$ & $9(28.2)$ \\
Total & 131 & 32 \\
\hline
\end{tabular}

AOCLF, acute-on-chronic liver failure; ALF, acute liver failure. Chi-square $p$ between groups: 0.0043 .

In the overall cohort, the majority of patients were female ( 99 female vs. 64 male). The proportion of male patients was significantly higher in AOCLF $(21 / 32 ; 65 \%)$ than in the ALF subgroup, $44 / 131$ patients were male $(34 \% ; p=0.0008)$. The mean age of all patients included in the study was $41.6 \pm$ 16.5. Patients with AOCLF were significantly older than patients with ALF $(50.3 \pm 15.1$ vs. $39.8 \pm 16.2, p=0.0008)$. Patients with AOCLF also had a significantly higher BMI (27.5 \pm 5.1 vs. $24.5 \pm 6.2, p=0.0014$; Table 1; Fig. 2 c).
BMI was significantly higher in AOCLF patients (c). Shown are scatter dot plots with mean and SEM. Statistical significance was determined by Mann-Whitney U Test.

\section{Patients with a Pre-Existing Liver Damage Show \\ Distinct Different Laboratory Parameters}

The serum concentrations of AST and ALT were strongly elevated above the normal range (male patients $50 \mathrm{U} / \mathrm{I}$, female $35 \mathrm{U} / \mathrm{I}$ ) in both ALF and AOCLF (Fig. 1). However, the mean AST was significantly lower in AO$\operatorname{CLF}(1,863 \pm 2,915)$ than in ALF patients $(2,882 \pm 3,339$; $p=0.01$ ). A similar relation was found for ALT (ALF: $3,206 \pm 3,382$; AOCLF: $1,662 \pm 2,125 ; p=0.001)$.

The markers for overall cell death (M65) and for apoptosis (M30) did not show significant differences between ALF and AOCLF, though serum levels were nominally higher in patients with ALF (online suppl. Fig. 1a, b). A similar observation was made for thrombocyte count, with slightly higher numbers in ALF, though no significant difference between the groups was found ( $p=0.39$; online suppl. Fig. 1c).

\section{Patients with an AOCLF have Different Etiology-}

Based Reasons for the Acute Insult

To identify a possible influence of the acute insult, etiologies causing ALF or AOCLF in the presented cohort were compared (Table 3). Within the ALF group,
Gottlieb/Kottmann/Manka/Bedreli/ Hadem/Bechmann/Sowa/Gerken/Canbay 
Table 4. Parameters separating survived and deceased patients within liver failure groups (ALF/AOCLF)

\begin{tabular}{|c|c|c|c|c|}
\hline Parameter & \multicolumn{2}{|l|}{$\mathrm{ALF}$} & \multicolumn{2}{|l|}{ AOCLF } \\
\hline BMI & $24.04 \pm 6.03$ & $27.58 \pm 6.5^{*}$ & $26.62 \pm 4.69$ & $31.22 \pm 5.42$ \\
\hline AST & $2,862 \pm 3,252$ & $2,988 \pm 3,834$ & $2,045 \pm 2,917$ & $1,320 \pm 3,039$ \\
\hline ALT & $3,344 \pm 3,537$ & $2,493 \pm 2,354$ & $1,976 \pm 2,260$ & $762 \pm 1,430^{*}$ \\
\hline $\mathrm{AP}$ & $149.8 \pm 114.8$ & $203.3 \pm 80.35^{* *}$ & $186.7 \pm 109$ & $147.1 \pm 49.31$ \\
\hline$\gamma \mathrm{GT}$ & $173.7 \pm 180.8$ & $350.7 \pm 401.4^{* * *}$ & $300.4 \pm 380.3$ & $196.1 \pm 90.46$ \\
\hline Phosphate & $3.28 \pm 1.14$ & $5.15 \pm 2.91^{* *}$ & $3.33 \pm 1.9$ & $3.9 \pm 1^{*}$ \\
\hline M65 & $9,432 \pm 10,056$ & $15,398 \pm 17,461$ & $6,627 \pm 5,777$ & $5,853 \pm 7,826$ \\
\hline M30 & $4,278 \pm 5,006$ & $6,949 \pm 9,192$ & $3,446 \pm 5,217$ & $2,049 \pm 1,916$ \\
\hline
\end{tabular}

${ }^{*} p<0.05 ; * *<0.01 ;{ }^{* * *} p<0.0001$ each vs. survival of the respective liver failure group by $t$ test 2 -way ANOVA.

ALF, acute liver failure; AOCLF, acute-on-chronic liver failure; BMI, body mass index; AST, aspartate aminotransferase; ALT, alanine aminotransferase; GLDH, glutamate dehydrogenase; AP, alcalic phosphatase; $\gamma \mathrm{GT}$, $\gamma$-glutamyltransferase; M65, surrogate cell death marker (epithelial cells); M30, surrogate apoptosis marker (epithelial cells); INR, international normalized ratio; MELD, model for end-stage liver disease; MMELD, CK18-modified MELD.

the most common acute insults were caused by pharmacological-toxic effects $(48 / 131 ; 27$ acetaminophenrelated), acute viral infections $(36 / 131)$ or remained unknown (21/131). Sixteen of the 131 ALF patients had an autoimmune-based liver failure. Ten cases were combined in the category of other cause (online suppl. Table 2). In contrast, the most common acute insults leading to AOCLF were acute viral infections (12/32) and "other causes" (9/32; online suppl. Table 1$)$. The remaining causes were pharmacological-toxic (4), unknown (4) and autoimmune (3). The distribution of acute etiologies causing liver failure were significantly different between ALF and AOCLF (chi-square $p=$ 0.0043).

\section{Patients with AOCLF and Higher Levels of Liver}

Enzymes have a Better Chance to Survive and to

\section{Undergo Spontaneous Remission}

As we were interested if the main outcomes - survival or remission - after ALF and AOCLF were different, both patient groups were separated either by survival (survived/deceased) or by remission (spontaneous remission, SR; non-spontaneous remission, including deceased and liver transplantation, NSR). The relative frequency of survival (ALF: 110 / 84 \%; AOCLF: 24/75\%) or SR (ALF:
88/67\%; AOCLF: 22/69\%) did not differ between ALF and AOCLF. While clinical outcome was not different between the groups analyzed, potential specific risk factors indicate adverse outcome in ALF or AOCLF. In the ALF group, significant differences between survivors and non-survivors were found for age, BMI, MELD, M30modified MELD, bilirubin, AP, $\gamma \mathrm{GT}$, INR, creatinine, and phosphate (all higher/elevated in deceased patients; Table 4). In the AOCLF group, significantly higher MELD and modified MELD, creatinine, and phosphate as well as lower ALT were found in deceased patients (Table 4). ALF patients with SR exhibited significantly lower age, MELD, modified MELD, bilirubin, AP, $\gamma$ GT, INR, and creatinine as well as higher ALT and AST (Table 5). AOCLF patients with SR had significantly higher ALT, AST, and GLDH as well as lower creatinine and MELD (Table $5)$.

\section{Discussion}

In this study, we describe a cohort of patients initially admitted to the hospital with a diagnosis of ALF. Upon retrospective examination, a relevant proportion had to be re-classified as AOCLF without liver cirrhosis. Almost 
Table 5. Parameters separating patients with spontaneous remission from patients with non-spontaneous remission ${ }^{1}$ within liver failure groups (ALF/AOCLF)

\begin{tabular}{|c|c|c|c|c|}
\hline Parameter & \multicolumn{2}{|l|}{$\mathrm{ALF}$} & \multicolumn{2}{|l|}{ AOCLF } \\
\hline BMI & $23.98 \pm 6.20$ & $25.68 \pm 6.05$ & $26.53 \pm 4.89$ & $30.14 \pm 4.96$ \\
\hline AST & $3,132 \pm 3,197$ & $2,382 \pm 2,404^{*}$ & $2,141 \pm 3,020$ & $1,248 \pm 2,717^{*}$ \\
\hline ALT & $3,603 \pm 3,601$ & $3,593 \pm 2,754^{*}$ & $2,042 \pm 2,325$ & $865.2 \pm 1,412^{*}$ \\
\hline $\mathrm{AP}$ & $153.7 \pm 125.9$ & $171.2 \pm 74.74^{* *}$ & $181.4 \pm 107$ & $168.3 \pm 82.5$ \\
\hline$\gamma \mathrm{GT}$ & $173 \pm 192.2$ & $265.9 \pm 309.3^{* *}$ & $316 \pm 394.8$ & $184.4 \pm 87.52$ \\
\hline Phosphate & $3.15 \pm 0.98$ & $4.32 \pm 2.58$ & $3.35 \pm 1.96$ & $3.67 \pm 0.93$ \\
\hline M65 IU & $8,784 \pm 9,368$ & $13,567 \pm 14,790$ & $6,944 \pm 5,915$ & $5,447 \pm 7,237$ \\
\hline M30 IU & $4,214 \pm 5,014$ & $5,674 \pm 7,304$ & $3,628 \pm 5,406$ & $1,936 \pm 1,774$ \\
\hline
\end{tabular}

\footnotetext{
${ }^{1}$ Non-spontaneous remission $=$ transplantation and/or death.

${ }^{*} p<0.05$; $^{* *} p<0.01{ }^{* * *} p<0.0001$ each vs. survival of the respective liver failure group by Mann-Whitney U test (due to nonnormally distributed data for all parameters).

ALF, acute liver failure; AOCLF, acute-on-chronic liver failure; BMI, body mass index; AST, aspartate aminotransferase; ALT, alanine aminotransferase; GLDH, glutamate dehydrogenase; AP, alcalic phosphatase; $\gamma \mathrm{GT}, \gamma$-glutamyltransferase; M65, surrogate cell death marker (epithelial cells); M30, surrogate apoptosis marker (epithelial cells); INR, international normalized ratio; MELD, model for end-stage liver disease; MMELD, CK18-modified MELD.
}

one-fifth (19.6\%) of the initial ALF cohort had a pre-existing liver damage, which had not been detected previously. None of these had cirrhosis yet nor a history of a prior decompensation of the liver function. While the clinical outcome of ALF and AOCLF did not differ in this study, each group exhibited difference in the etiology of acute injury leading to liver failure and in possible risk factors for outcome.

The EASL as well as the APASL each proposed a definition of AOCLF, which unfortunately are not in full accordance. This unclear terminology complicates the clinical definition of this entity and discussions in research on this topic. The EASL definition of AOCLF only comprises patients with cirrhosis, including those with previous decompensation. In contrast, Sarin et al. [12] have been trying to broaden the definition by including preexisting liver cirrhosis and also patients with a pre-existing liver damage with no signs of cirrhosis into AOCLF. In our opinion, both definitions are not clear enough for routine use. Thus, we propose to define patients with liver failure, with a pre-existing liver damage (independent of the cause, e.g., fatty liver disease, chronic viral hepatitis, etc) with no cirrhosis and no prior history of decompen- sation as AOCLF. Patients with cirrhosis, independent of decompensation events, would be grouped as acute-oncirrhosis liver failure (AOC).

In the present study, the clinical presentation of ALF and AOCLF differed to a significant extent. Patients with AOCLF were more often male, older, and had a higher BMI compared to patients with ALF. We had previously reported that patients with ALF and an actually pre-existing liver disease have higher BMI compared to patients with ALF and no undetected liver disease [18]. Additionally, AOCLF patients in our cohort had lower serum liver injury markers. While the outcome of AOCLF was not impaired compared to ALF, lower AST and ALT have been shown to be associated with adverse outcome in ALF [18]. This has been confirmed for relatively lower markers of cell death and apoptosis, which has been interpreted as a higher amount of intact parenchymal liver cells present before acute injury [19], which can release large amounts of the respective protein into circulation. In agreement with these data, cell death markers were lower in AOCLF in the present study, though significance was not reached. It could be speculated that surrogate markers of other cell death modes, that is, HMGB1, 
may also facilitate separation of ALF and AOCLF. The present analysis was limited to M65 and M30. Moreover, thrombocyte counts did not differ significantly between ALF and AOCLF patients. This observation clearly demonstrates that acute liver injury can occur in at least three settings. A true ALF (without any pre-existing liver disease), an acute-on-chronic failure (with pre-existing liver disease but without cirrhotic alterations), and acuteon-cirrhotic liver failure (with pre-existing cirrhosis), the latter presenting with much more sever disease course. Nevertheless, AOCLF should be clearly distinguished from ALF and AOC as these should probably be treated differently. However, larger studies will be required to determine the ideal management of these complicated cases.

The acute injury initiating liver failure differed significantly between ALF and AOCLF. Pharmacologic-toxic, acute viral, and unknown causes were the main causes for ALF, while in AOCLF acute viral infections and rare causes dominated. Unclear cases of ALF in older patients with viral component or a rare cause for ALF should be evaluated for a pre-existing liver injury.

Current definitions of AOCLF are highly incongruous, resulting in very different clinical outcomes observed in AOCLF cohorts. According to the European and American consensus definition of ACLF, which restricts the pre-existing entity to (undiagnosed) liver cirrhosis and involves extrahepatic organ failure, the 28 -mortality is up to $75 \%$ [20]. This is not surprising given the unfavorable requirements to define ACLF. In the present study, a much lower mortality rate was observed in AOCLF patients, which did not differ significantly from ALF. The slightly worse outcome of AOCLF patients can be explained, for example, with a higher BMI. It has been shown that recovery from hepatic ATP depletion is severely impaired in patients with obesityrelated NASH [21]. As the basic clinical presentation differed between the groups, we aimed to identify specific risk factors for adverse outcome, though the rate of adverse outcomes may not be different. MELD and the M30-modified MELD were risk factors for adverse outcome in both ALF and AOCLF. Thus, the MELD and the modified MELD are relevant predictors for liver failure outcome, even with an underlying chronic (non-cirrhotic) liver disease. Nevertheless, for outcome in ALF further risk factors were higher BMI, higher bilirubin, AP, $\gamma \mathrm{GT}$, and INR, which were not significantly different between the AOCLF outcome groups. Conversely, low ALT was a predictor for mortality and low GLDH for non-spontaneous remission in AOCLF but not in

Define ALF Patients with Pre-Existing

Liver Disease without Signs of Cirrhosis
ALF. These findings suggest that ALF and AOCLF represent clinically separate entities, despite similar outcomes.

According to the findings presented here, we think it is important to agree on an internationally consistent definition based on which patients should be considered to have an AOCLF with clear and exact criteria to separate them from patients with ALF and those with acuteon-cirrhosis liver failure, as the latter has a fundamentally higher mortality $[14,22]$. When a universal definition for these three entities has been agreed upon, multi-center studies with larger patient numbers should be carried out to get a better understanding of all types of liver failure. This will allow earlier correct diagnosis and timely treatment and therapy, as necessary. This will also influence the decision-making process, whether to evaluate these patients for liver transplantation or help prevent AOC.

There are several limitations of the present study. Due to the retrospective nature, not all datasets are complete for all patients. Apart from this, ultrasound assessment of liver parenchyma was done in a small subset of this cohort, barring relevant statistical evaluation. As liver biopsy is not routinely performed in patients with acute liver injury, no histological samples were available for this study. The group sizes for the different entities were not evenly split, causing a difference in data variation. This was accounted for by predominantly applying non-parametric tests, which are more robust in this situation.

In summary, we could show significant clinical differences between a true ALF compared to patients with preexisting liver damage without liver cirrhosis suffering from an AOCLF. Patients with AOCLF were older, had a higher BMI, and were more often male. Serum markers to predict outcome differed between ALF and AOCLF. The Meld-score would be applicable for both patients with ALF and AOCLF. The overall prognosis for remission and survival was similar between ALF and AOCLF, though further studies are warranted to identify specific risk factors and optimal therapy for both entities.

\section{Disclosure Statement}

The authors declare no conflict of interest.

\section{Financial support}

No funding. 


\section{References}

1 Sowa JP, Gerken G, Canbay A: Acute liver failure - it's just a matter of cell death. Dig Dis (Basel, Switzerland) 2016;34:423-428.

2 Schiodt FV, Davern TJ, Shakil AO, et al: Viral hepatitis-related acute liver failure. Am J Gastroenterol 2003;98:448-453.

3 Shakil AO, Kramer D, Mazariegos GV, et al: Acute liver failure: clinical features, outcome analysis, and applicability of prognostic criteria. Liver Transpl 2000;6:163-169.

4 Manka P, Verheyen J, Gerken G, et al: Liver failure due to acute viral hepatitis (A-E). Visc Med 2016;32:80-85.

5 Ruhl CE, Everhart JE: Determinants of the association of overweight with elevated serum alanine aminotransferase activity in the United States. Gastroenterology 2003;124:71-79.

6 Younossi ZM, Koenig AB, Abdelatif D, et al: Global epidemiology of nonalcoholic fatty liver disease-Meta-analytic assessment of prevalence, incidence, and outcomes. Hepatology 2016;64:73-84.

7 Sydor S, Gu Y, Schlattjan M, et al: Steatosis does not impair liver regeneration after partial hepatectomy. Lab Invest 2013;93:20-30.

8 Canbay A, Jochum C, Bechmann LP, et al: Acute liver failure in a metropolitan area in Germany: a retrospective study (2002-2008). Zeitschrift fur Gastroenterologie 2009;47: 807-813.
9 Kooby DA, Fong Y, Suriawinata A, et al: Impact of steatosis on perioperative outcome following hepatic resection. J Gastrointest Surg 2003;7:1034-1044.

10 Verran D, Kusyk T, Painter D, et al: Clinical experience gained from the use of 120 steatotic donor livers for orthotopic liver transplantation. Liver Transpl 2003;9:500-505.

11 van den Berg EH, Douwes RM, Meijer VE de, et al: Liver transplantation for NASH cirrhosis is not performed at the expense of major post-operative morbidity. Dig Liver Dis 2018; 50:68-75.

12 Sarin SK, Choudhury A: Acute-on-chronic liver failure: terminology, mechanisms and management. Nat Rev Gastroenterol Hepatol 2016;13:131-149.

13 Abbas Z, Shazi L: Pattern and profile of chronic liver disease in acute on chronic liver failure. Hepatol Int 2015;9:366-372.

14 Jalan R, Saliba F, Pavesi M, et al: Development and validation of a prognostic score to predict mortality in patients with acute-on-chronic liver failure. J Hepat 2014;61:1038-1047.

15 Arroyo V, Jalan R: Acute-on-chronic liver failure: definition, diagnosis, and clinical characteristics. Semin Liver Dis 2016;36:109116.

16 Sarin SK, Kedarisetty CK, Abbas Z, et al: Acute-on-chronic liver failure: consensus recommendations of the Asian Pacific Association for the Study of the Liver (APASL) 2014. Hepatol Int 2014;8:453-471.

17 Bechmann LP, Jochum C, Kocabayoglu P, et al: Cytokeratin 18-based modification of the MELD score improves prediction of spontaneous survival after acute liver injury. J Hepatol 2010;53:639-647.

18 Canbay A, Gieseler RK, Gores GJ, et al: The relationship between apoptosis and non-alcoholic fatty liver disease: an evolutionary cornerstone turned pathogenic. Zeitschrift fur Gastroenterologie 2005;43:211-217.

19 Volkmann X, Anstaett M, Hadem J, et al: Caspase activation is associated with spontaneous recovery from acute liver failure. Hepatology 2008;47:1624-1633.

20 Bruns T, Stallmach A: Akut-auf-chronisches Leberversagen: neue definition eines komplexen krankheitsbildes. Deutsche medizinische Wochenschrift (1946) 2016;141: 798-802.

21 Cortez-Pinto H, Chatham J, Chacko VP, et al: Alterations in liver ATP homeostasis in human nonalcoholic steatohepatitis: a pilot study. JAMA 1999;282:1659-1664.

22 Canbay A, Tacke F, Hadem J, et al: Acute liver failure: a life-threatening disease. Deutsches Arzteblatt international 2011;108:714720. 


\section{DuEPublico}

\section{Duisburg-Essen Publications online}

DESISEBN R G

offen im Denken

This text is made available via DuEPublico, the institutional repository of the University of Duisburg-Essen. This version may eventually differ from another version distributed by a commercial publisher.

DOI: $\quad 10.1159 / 000492869$

URN: urn:nbn:de:hbz:464-20201116-164239-9

This publication is with permission of the rights owner freely accessible due to an Alliance licence and a national licence (funded by the DFG, German Research Foundation) respectively.

All rights reserved. 